\title{
ADJOINT OPERATORS IN LIE ALGEBRAS AND THE CLASSIFICATION OF SIMPLE FLEXIBLE LIE-ADMISSIBLE ALGEBRAS
}

\author{
BY \\ SUSUMU OKUBO AND HYO CHUL MYUNG ${ }^{1}$
}

\begin{abstract}
Let $\mathfrak{A}$ be a finite-dimensional flexible Lie-admissible algebra over an algebraically closed field $F$ of characteristic 0 . It is shown that if $\mathfrak{A}^{-}$is a simple Lie algebra which is not of type $A_{n}(n>2)$ then $\mathfrak{A}$ is a Lie algebra isomorphic to $\mathfrak{A}^{-}$, and if $\mathfrak{A}^{-}$is a simple Lie algebra of type $A_{n}(n>2)$ then $\mathfrak{A}$ is either a Lie algebra or isomorphic to an algebra with multiplication $x * y=\mu x y+(1-\mu) y x-$ $(1 /(n+1)) \operatorname{Tr}(x y) I$ which is defined on the space of $(n+1) \times(n+1)$ traceless matrices over $F$, where $x y$ is the matrix product and $\mu \neq \frac{1}{2}$ is a fixed scalar in $F$. This result for the complex field has been previously obtained by employing an analytic method. The present classification is applied to determine all flexible Lie-admissible algebras $\mathfrak{A}$ such that $\mathfrak{A}^{-}$is reductive and the Levi-factor of $\mathfrak{A}^{-}$is simple. The central idea is the notion of adjoint operators in Lie algebras which has been studied in physical literature in conjunction with representation theory.
\end{abstract}

1. Introduction. For an algebra $\mathfrak{A}$ over a field $F$, denote by $\mathfrak{A}^{-}$the algebra defined on the vector space $\mathfrak{A}$ with multiplication given by the commutation $[x, y]=x y-y x$. Then $\mathfrak{A}$ is said to be Lie-admissible if $\mathfrak{A}^{-}$is a Lie algebra, that is, $\mathfrak{A}^{-}$satisfies the Jacobi identity $[x,[y, z]]+[y,[z, x]]+[z,[x, y]]=0$. If a Lie-admissible algebra $\mathfrak{A}$ satisfies the flexible law $(x y) x=x(y x)$ then it is called flexible Lie-admissible. Applications of Lie-admissible algebras in physics have been pointed out by a number of physicists and arise in both the classical and quantum mechanics. For this, the reader is referred, for example, to Santilli [17].

All algebras considered in this paper are finite-dimensional over a field $F$. Let $\mathfrak{L}$ be a Lie algebra over $F$ and let $\left\{x_{1}, x_{2}, \ldots, x_{N}\right\}$ be an ordered basis for $\mathfrak{Q}$ with multiplication table

$$
\left[x_{\mu}, x_{\nu}\right]=\sum_{\lambda=1}^{N} f_{\mu \nu}^{\lambda} x_{\lambda}
$$

where $f_{\mu \nu}^{\lambda} \in F$ are the structure constants of $\mathfrak{L}$. If $\rho$ is a representation of $\mathfrak{L}$ acting on a $d$-dimensional vector space $\mathfrak{M}$ over $F$, denote by $\operatorname{gl}(\mathfrak{M})$ and $\operatorname{gl}(d, F)$ the general linear (Lie) algebras of linear transformations on $\mathfrak{M}$ and $d \times d$ matrices over $F$, respectively. We often identify $\mathfrak{g l}(\mathfrak{M})$ with $\mathfrak{g l}(d, F)$.

Received by the editors March 20, 1980; presented to the Society, April 18, 1979, under the title Adjoint operators and simple flexible Lie-admissible algebras.

AMS (MOS) subject classifications (1970). Primary 17A20, 17B05, 17B10, 17B20, $17 \mathrm{~B} 25$.

Key words and phrases. Lie algebra, flexible Lie-admissible algebra, adjoint operator, weight, highest adjoint weight, Weyl basis, reductive Lie algebra, adjoint dimension, representation.

'The first author was supported in part by the U. S. Department of Energy under Contract Number EY-76-C-02-3065. 
Definition 1.1. Let $\rho: \mathfrak{L} \rightarrow \mathfrak{g l}(\mathfrak{M})$ (or $\mathfrak{g l}(d, F)$ ) be a representation of $\mathfrak{L}$. Then, a linear mapping $S: \mathfrak{I} \rightarrow \mathfrak{g l}(\mathfrak{M})$ (or $\mathfrak{g l}(d, F)$ ) is called an adjoint (or vector) operator of $\mathfrak{Q}$ in $\rho$, if $S$ satisfies the commutation relation

$$
S([x, y])=[\rho(x), S(y)] .
$$

Denote by $V_{\rho}(\mathfrak{L}, \mathfrak{M})$ or $V_{\rho}(\mathfrak{R}, d)$ the vector space over $F$ of all adjoint operators of $\mathfrak{L}$ in $\rho$. It is easily seen that $V_{\rho}(\mathfrak{R}, \mathfrak{M})$ is isomorphic to the vector space of all ordered $N$-tuples $T=\left(T_{1}, T_{2}, \ldots, T_{N}\right), T_{\lambda} \in \mathfrak{g l}(\mathfrak{M})$, which satisfy the relations $\left[\rho\left(x_{\mu}\right), T_{\nu}\right]=\sum_{\lambda=1}^{N} f_{\mu \nu}^{\lambda} T_{\lambda}$. In particular, if $\rho=$ ad, the adjoint representation of $\mathfrak{L}$, we write $V_{0}(\mathfrak{R})$ for $V_{\rho}(\mathfrak{R}, \mathfrak{M})$. Since $\rho \in V_{\rho}(\mathfrak{L}, \mathfrak{M})$, we have $\operatorname{dim} V_{\rho}>1$, assuming $\rho \neq 0$. The notion of adjoint operators was first introduced by Wigner [20] for the Lie algebra of the SU(2) group and the general case has been studied in physical literature, recently by Okubo [12], [13]. For an irreducible representation $\rho$ of a simple Lie algebra $\mathfrak{L}$ over the complex field $F$, the enumeration of $\operatorname{dim} V_{\rho}(\mathfrak{Q}, \mathfrak{M})$ (called here the adjoint dimension theorem) has been established by Okubo [13, p. 2392] in conjunction with representation theory by invoking the transcendental method of the Wigner-Eckart theorem [16, p. 474]. Consequently, the method employed in [13] is not algebraic. In particular, the adjoint dimension theorem implies that $\operatorname{dim} V_{0}(\mathfrak{R})=1$ or 2 . This result has been recently utilized by Okubo and Myung [15] to classify flexible Lie-admissible algebras $\mathfrak{A}$ such that $\mathfrak{U}^{-}$is a simple Lie algebra over the complex field. Specifically, it is shown in [15] that if $\mathfrak{A}^{-}$ is a simple Lie algebra which is not of type $A_{n}(n \geqslant 2)$ then $\mathfrak{A}$ is itself a Lie algebra isomorphic to $\mathfrak{A}^{-}$, and if $\mathfrak{U}^{-}$is of type $A_{n}(n \geqslant 2)$ then $\mathfrak{A}$ is either a Lie algebra or isomorphic to an algebra with multiplication given by

$$
x * y=\mu x y+(1-\mu) y x-\operatorname{Tr}(x y) I /(n+1),
$$

which is defined on the vector space of $(n+1) \times(n+1)$ traceless matrices over the complex field, where $x y$ is the matrix product, $\mu \neq \frac{1}{2}$ is a complex number and $I$ is the unit matrix.

The purpose of this paper is two-fold. First, we prove the adjoint dimension theorem for an arbitrary algebraically closed field of characteristic 0 in a purely algebraic manner which is based on representation theory (Theorem 3.1). Next, we obtain the aforementioned classification of flexible Lie-admissible algebras over an algebraically closed field of characteristic 0 (Theorem 4.3).

The present result essentially completes the classification of flexible Lie-admissible algebras $\mathfrak{A}$ with $\mathfrak{A}^{-}$semisimple in characteristic 0 which was proposed by Albert [1] in 1948 (Corollary 4.1). As an immediate consequence of this we obtain the result of Laufer and Tomber [9] that if, in addition, $\mathfrak{A}$ is power-associative then $\mathfrak{A}$ is a Lie algebra isomorphic to $\mathfrak{U}^{-}$. Thus the simple algebras associated with type $A_{n}(n \geqslant 2)$ yield a new family of simple flexible algebras which are neither power-associative nor have a unit element. The classification of these algebras in prime characteristic is a long-standing open problem as it is for Lie algebras. The present classification can be applied to determine all flexible Lie-admissible algebras $\mathfrak{A}$ with $\mathfrak{A}^{-}$reductive where the Levi-factor of $\mathfrak{A}^{-}$is a simple Lie algebra (Theorem 5.1). 
The central idea is to introduce the highest adjoint weight associated with an adjoint operator and the highest weight of an irreducible representation of a simple Lie algebra. The crucial fact is that an adjoint operator is completely determined by its highest adjoint weight (Theorem 2.1).

2. Highest adjoint weights of adjoint operators. We retain notations in $\S 1$. Henceforth we assume that $\mathfrak{L}$ denotes a finite-dimensional simple Lie algebra over an algebraically closed field $F$ of characteristic 0 and all representations $\rho$ : $\mathfrak{L} \rightarrow \mathfrak{g l}(\mathfrak{M})$ are nonzero finite-dimensional and irreducible. Hence $\rho$ is faithful and we identify $\rho(x)=x, x \in \mathfrak{L}$, so $\mathfrak{M}$ is regarded as an irreducible $\mathfrak{R}$-module via $\rho(x) v=x v, v \in \mathfrak{M}, x \in \mathfrak{R}$.

Let $\mathfrak{G}$ be a fixed Cartan subalgebra of $\mathfrak{L}$ and (, ) be the Killing form of $\mathfrak{L}$. Let $\mathfrak{L}=\mathfrak{Q}+\sum_{\alpha \neq 0} \mathfrak{L}_{\alpha}$ be the Cartan decomposition of $\mathfrak{L}$ relative to $\mathfrak{Q}$, where $\mathfrak{L}_{\alpha}$ is the root space of $\mathbb{R}$ for a root $\alpha \neq 0$. We adopt a lexicographic ordering of the roots determined by a fundamental system of roots $\pi=\left\{\alpha_{1}, \alpha_{2}, \ldots, \alpha_{n}\right\}$ where $n=$ rank $\mathfrak{R}$. Let $h_{1}, h_{2}, \ldots, h_{n}$ be the basis of $\mathfrak{E}$ corresponding to $\pi$, and let $\psi \rightarrow h_{\psi}$ be the isomorphism of $\mathfrak{Q}^{*}$ (the dual space of $\mathfrak{Q}$ ) to $\mathfrak{Q}$ defined by $\left(h, h_{\psi}\right)=\psi(h), h \in \mathfrak{Q}$. Then for $\psi \in \mathfrak{S}^{*}$,

$$
\begin{gathered}
h_{\psi}=\sum_{j=1}^{n} \psi^{j} h_{j}, \quad \psi^{j} \in F, \\
(\psi, \phi) \equiv\left(h_{\psi}, h_{\phi}\right)=\sum_{i=1}^{n} \psi^{i} \phi\left(h_{i}\right) .
\end{gathered}
$$

Since if $\alpha+\beta$ is a nonzero root then $\left[\mathfrak{R}_{\alpha}, \mathfrak{R}_{\beta}\right] \neq 0$, as in Jacobson [6, p. 147], one can find the following Weyl basis for $\mathfrak{R}$ with multiplication given by

$$
\begin{aligned}
{\left[h_{i}, h_{j}\right] } & =0, \quad i, j=1,2, \ldots, n, \\
{\left[h_{i}, e_{\alpha}\right] } & =\alpha\left(h_{i}\right) e_{\alpha}, \\
{\left[e_{\alpha}, e_{\beta}\right] } & =N_{\alpha, \beta} e_{\alpha+\beta}, \quad \alpha+\beta \neq 0, \\
{\left[e_{\alpha}, e_{-\alpha}\right] } & =\sum_{i=1}^{n} \alpha^{i} h_{i},
\end{aligned}
$$

where $\alpha, \beta$ represent nonzero roots and $N_{\alpha, \beta} \in F$ satisfies

$$
N_{\alpha, \beta}=N_{-\alpha,-\beta} \text {. }
$$

Denote the basis for $\mathfrak{L}$ in (2.2) by a short-hand notation $\left\{x_{\mu}\right\}=\left\{h_{i}, e_{\alpha}, e_{-\alpha}\right\}$ in a fixed order with $\alpha$ representing all positive roots of $\mathfrak{Q}$. Accordingly, we denote by $\left(S_{\mu}\right)=\left(H_{i}, E_{\alpha}, E_{-\alpha}\right)$ an adjoint operator in $V_{\rho}(\mathfrak{L}, \mathfrak{M})$. Thus, by Definition 1.1, $E_{\alpha}$, $E_{-\alpha}$ for $\alpha>0$, and $H_{i}(i=1,2, \ldots, n)$ are elements in $\mathfrak{g l}(\mathfrak{M})$ satisfying the commutation relations

$$
\begin{aligned}
{\left[h_{j}, H_{k}\right] } & =0, \\
{\left[h_{i}, E_{\alpha}\right] } & =\left[H_{i}, e_{\alpha}\right]=\alpha\left(h_{i}\right) E_{\alpha}, \\
{\left[h_{i}, E_{-\alpha}\right] } & =\left[H_{i}, e_{-\alpha}\right]=-\alpha\left(h_{i}\right) E_{-\alpha}, \\
{\left[e_{\alpha}, E_{\beta}\right] } & =N_{\alpha, \beta} E_{\alpha+\beta}, \quad \alpha+\beta \neq 0, \\
{\left[e_{\alpha}, E_{-\alpha}\right] } & =\left[E_{\alpha}, e_{-\alpha}\right]=\sum_{i} \alpha^{i} H_{i} .
\end{aligned}
$$


Let $\Lambda$ be the highest weight of $\mathfrak{S}$ in the irreducible $\mathfrak{L}$-module $\mathfrak{M}$. Let $v^{+} \in \mathfrak{M}$ be a maximal (or highest weight) vector of $\Lambda$. Thus $h v^{+}=\Lambda(h) v^{+}, h \in \mathfrak{S}$, and $e_{\alpha} v^{+}=0, \alpha>0$. Let $\left(H_{i}, E_{\alpha}, E_{-\alpha}\right) \in V_{\rho}(\mathfrak{L}, \mathfrak{M})$. Since $\left[h, H_{j}\right]=0$ for $h \in \mathfrak{Q}$,

$$
h\left(H_{j} v^{+}\right)=H_{j}\left(h v^{+}\right)=\Lambda(h) H_{j} v^{+}, \quad h \in \mathfrak{B} .
$$

Hence $H_{j} v^{+}, j=1,2, \ldots, n$, is a maximal vector of $\Lambda$ in $\mathfrak{M}[6$, p. 215] and since a maximal vector is unique up to scalar multiple, we have

$$
H_{j} v^{+}=\Gamma_{j} v^{+}, \quad j=1,2, \ldots, n,
$$

where $\Gamma_{j} \in F$ is uniquely determined by $H_{j}$. This leads to

Definition 2.1. For an adjoint operator $\left(S_{\mu}\right)=\left(H_{i}, E_{\alpha}, E_{-\alpha}\right)$ in $V_{\rho}(\mathfrak{L}, \mathfrak{M})$, the highest adjoint weight $\Gamma$ of $\left(S_{\mu}\right)$ for $\mathfrak{G}$ in $\mathfrak{M}$ is defined as the element $\Gamma \in \mathfrak{S}^{*}$ such that $\Gamma\left(h_{i}\right)=\Gamma_{i}(i=1,2, \ldots, n)$ with $\Gamma_{i} \in F$ in (2.5). We call $\left(H_{i}\right)=$ $\left(H_{1}, \ldots, H_{n}\right)$ the Cartan part of $\left(S_{\mu}\right)$ relative to $\mathfrak{Q}$.

LemMa 2.1. Let $\left(H_{i}, E_{\alpha}, E_{-\alpha}\right) \in V_{\rho}(\mathfrak{R}, \mathfrak{M})$. Then, for any positive root $\alpha, E_{\alpha} v^{+}=$ 0 , and $E_{-\alpha} v^{+}$is either 0 or a weight vector in $\mathfrak{M}$ with weight $\Lambda-\alpha$.

Proof. In view of (2.4), we have

$$
h\left(E_{\alpha} v^{+}\right)=E_{\alpha} h v^{+}+\alpha(h) E_{\alpha} v^{+}=(\Lambda(h)+\alpha(h)) E_{\alpha} v^{+}
$$

for $h \in \mathfrak{Q}$. Thus if $E_{\alpha} v^{+} \neq 0, \Lambda+\alpha$ is a weight and this is impossible since $\Lambda$ is the highest weight and $\alpha>0$. Similarly, one gets $h\left(E_{-\alpha} v^{+}\right)=(\Lambda-\alpha)(h) E_{-\alpha} v^{+}$ and this implies the second part.

LEMMA 2.2. Let $\Gamma$ be the highest adjoint weight of an adjoint operator $\left(H_{i}, E_{\alpha}, E_{-\alpha}\right)$ in $V_{\rho}(\mathfrak{R}, \mathfrak{M})$. If $(\alpha, \Lambda)=0$ for a positive root $\alpha$ then $(\alpha, \Gamma)=0$.

Proof. Let $j, k$ be the least nonnegative integers such that $\Lambda-(k+1) \alpha$ and $\Lambda+(j+1) \alpha$ are not weights. We have the weight string of $\alpha$ through $\Lambda: \Lambda-k \alpha$, $\Lambda-(k-1) \alpha, \ldots, \Lambda, \Lambda+\alpha, \ldots, \Lambda+j \alpha$, all consisting of weights [6, p. 113], and $2(\Lambda, \alpha) /(\alpha, \alpha)=k-j$. Since $\Lambda$ is highest and $\alpha>0$, we must have $j=0$. Therefore, if $(\Lambda, \alpha)=0$ then $k=0$ and $\Lambda-\alpha$ is not a weight. This, in view of Lemma 2.1, implies $E_{-\alpha} v^{+}=0$ and so by (2.1) and (2.4) one gets

$$
\begin{aligned}
0 & =e_{\alpha} E_{-\alpha} v^{+}=\left(E_{-\alpha} e_{\alpha}+\left[e_{\alpha}, E_{-\alpha}\right]\right) v^{+} \\
& =\sum_{i} \alpha^{i} H_{i} v^{+}=\left(\sum_{i} \alpha^{i} \Gamma_{i}\right) v^{+} \\
& =\sum_{i} \alpha^{i} \Gamma\left(h_{i}\right) v^{+}=(\alpha, \Gamma) v^{+} . \square
\end{aligned}
$$

If $U(\mathfrak{L})$ denotes the universal enveloping algebra of $\mathfrak{L}$ then $\mathfrak{M}$ is regarded as a $U(\mathfrak{R})$-module and $U(\mathfrak{L}) v^{+}=\mathfrak{M}\left[5\right.$, p. 108]. Suppose that $S_{\mu} v^{+}=0$ for $\left(S_{\mu}\right) \in$ $V_{\rho}(\mathfrak{R}, \mathfrak{M})$. Since $x_{\mu} S_{\nu}-S_{\nu} x_{\mu}=\Sigma_{\lambda} f_{\mu \nu}^{\lambda} S_{\lambda}$ where $f_{\mu \nu}^{\lambda}$ are the structure constants for $\mathfrak{R}$, we have $S_{\mu}\left(\mathfrak{L} v^{+}\right)=0$ for all $\mu$. Noting $\left[x y, S_{\mu}\right]=x\left[y, S_{\mu}\right]+\left[x, S_{\mu}\right] y$ for $x, y \in \mathfrak{L}$ and so $S_{\mu} x y v^{+}=0$, by induction one sees that $S_{\mu} U(\mathfrak{R}) v^{+}=S_{\mu} \mathfrak{M}=0$. Thus we have

Lemma 2.3. Let $\left(S_{\mu}\right) \in V_{\rho}(\mathfrak{Q}, \mathfrak{M})$. If $S_{\mu} v^{+}=0$ for all $\mu$ then $\left(S_{\mu}\right)=0$. 
THEOREM 2.1. Let $\Gamma$ be the highest adjoint weight of an adjoint operator $\left(S_{\mu}\right) \in$ $V_{\rho}(\mathfrak{R}, \mathfrak{M})$. If $\Gamma=0$ then $\left(S_{\mu}\right)=0$.

Proof. Let $\left(S_{\mu}\right)=\left(H_{i}, E_{\alpha}, E_{-\alpha}\right)$ and let $U\left(\mathfrak{L}_{-}\right)$be the subalgebra of $U(\mathfrak{L})$ generated by $\mathfrak{L}_{-} \equiv \Sigma_{\alpha>0} \mathfrak{L}_{-\alpha}$. We show that $\mathfrak{M}_{0} \equiv \Sigma_{\alpha>0} U\left(\mathfrak{L}_{-}\right) E_{-\alpha} \mathcal{v}^{+}$is an R-submodule of $\mathfrak{M}$.

To see $h \mathfrak{M}_{0} \subseteq \mathfrak{M}_{0}$ for $h \in \mathfrak{Q}$, by induction it suffices to observe that he $e_{-\alpha}=$ $\left[h, e_{-\alpha}\right]+e_{-\alpha} h=-\alpha(h) e_{-\alpha}+e_{-\alpha} h$ and $h E_{-\alpha}=\left[h, E_{-\alpha}\right]+E_{-\alpha} h=-\alpha(h) E_{-\alpha}$ $+E_{-\alpha} h$. For $\beta<0$, we clearly have $e_{\beta} \mathfrak{M}_{0} \subseteq \mathfrak{M}_{0}$. To show $e_{\beta} \mathfrak{M}_{0} \subseteq \mathfrak{M}_{0}$ for $\beta>0$, let $f$ be any element of $U\left(\mathfrak{L}_{-}\right)$. Since $v^{+}$is a maximal vector, we have

$$
e_{\beta} f E_{-\alpha} v^{+}=\left[e_{\beta}, f\right] E_{-\alpha} v^{+}+f\left[e_{\beta}, E_{-\alpha}\right] v^{+} \text {. }
$$

To see that $\left[e_{\beta}, f\right] E_{-\alpha} v^{+} \in \mathfrak{M}_{0}$, by induction one may assume that $f=e_{-\gamma}$ for $\gamma>0$. Set $v=\left[e_{\beta}, e_{-\gamma}\right] E_{-\alpha} v^{+}$. If $\beta-\gamma=0, v=h E_{-\alpha} v^{+}$for some $h \in \mathfrak{B}$ and $v \in \mathfrak{M}_{0}$ since $h \mathfrak{M}_{0} \subseteq \mathfrak{M}_{0}$. If $\beta-\gamma$ is a negative root then $v=N \beta_{-\gamma} e_{\beta-\gamma} E_{-\alpha} v^{+}$, so $v \in \mathfrak{M}_{0}$. Finally, let $\beta-\gamma$ be a positive root. Then

$$
v=N_{\beta,-\gamma} e_{\beta-\gamma} E_{-\alpha} v^{+}=N_{\beta,-\gamma}\left[e_{\beta-\gamma}, E_{-\alpha}\right] v^{+} .
$$

Therefore, in view of (2.6), it remains to verify $\left[e_{\beta}, E_{-\alpha}\right] v^{+} \in \mathfrak{M}_{0}$ for $\beta>0$. If $\beta-\alpha<0$ then $\left[e_{\beta}, E_{-\alpha}\right] v^{+}=N_{\beta,-\alpha} E_{-(\alpha-\beta)} v^{+} \in \mathfrak{M}_{0}$. On the other hand, if $\beta-\alpha>0,\left[e_{\beta}, E_{-\alpha}\right] v^{+}=N_{\beta,-\alpha} E_{\beta-\alpha} v^{+}=0$ by Lemma 2.1. For the remaining case of $\beta=\alpha$, we see

$$
\left[e_{\alpha}, E_{-\alpha}\right] v^{+}=\sum_{i} \alpha^{i} H_{i} v^{+}=(\alpha, \Gamma) v^{+}=0
$$

since $\Gamma=0$. Thus we have proved that $\mathfrak{M}_{0}$ is an $\mathfrak{L}$-submodule of $\mathfrak{M}$.

Since $\mathfrak{M}_{0}$ is spanned by weight vectors $E_{-\alpha} v^{+}, e_{-\beta} E_{-\alpha} v^{+}, \ldots$ with weights $\Lambda-\alpha, \Lambda-\alpha-\beta, \ldots$ which are lower than $\Lambda, \mathfrak{M}_{0}$ cannot contain $v^{+}$. This implies $\mathfrak{M}_{0}=0$ since $\mathfrak{M}$ is irreducible. In particular, $E_{-\alpha} v^{+}=0$ for $\alpha>0$ while $E_{\alpha} v^{+}=0$ by Lemma 2.1. Hence $S_{\mu} v^{+}=0$ for all $\mu$ since $H_{j} v^{+}=\Gamma_{j} v^{+}=0$, $j=1,2, \ldots, n$. This proves $\left(S_{\mu}\right)=0$ by Lemma 2.3 .

In view of Theorem 2.1 , an adjoint operator in $V_{\rho}(\mathfrak{L}, \mathfrak{R})$ is uniquely determined by its highest adjoint weight and so by its Cartan part. As an immediate consequence of Theorem 2.1, we have

Corollary 2.1. Let $S^{(j)}=\left(S_{\mu}^{(j)}\right), j=1,2, \ldots, p$, be adjoint operators in $V_{\rho}(\mathfrak{R}, \mathfrak{M})$ and let $\Gamma^{(j)}(j=1,2, \ldots, p)$ be the highest adjoint weight of $S^{(j)}$. If

$$
\sum_{j=1}^{p} c_{j} \Gamma^{(j)}=0, \quad c_{j} \in F,
$$

then $\sum_{j=1}^{p} c_{j} S^{(j)}=0$.

3. The adjoint dimension. Let $\mathfrak{L}$ and $\rho: \mathfrak{L} \rightarrow \mathfrak{g l}(\mathfrak{M})$ be the same as in $\S 2$. In this section we enumerate $\operatorname{dim} V_{\rho}(\mathfrak{R}, \mathfrak{M})$ in terms of the highest weight $\Lambda$ by utilizing the results established in $\S 2$. The present proof is purely algebraic in contrast to the case of the complex field which was established in [12], [13] by employing an analytic method. As we will see shortly, $\operatorname{dim} V_{\rho}(\mathfrak{L}, \mathfrak{M})$ depends on the simple Lie algebra $\mathfrak{L}$ as well as the irreducible representation $\rho$. 
Definition 3.1. $\operatorname{dim} V_{\rho}(\mathfrak{R}, \mathfrak{M})$ is called the adjoint dimension of $\mathfrak{L}$ in the representation $\rho: \mathfrak{L} \rightarrow \mathfrak{g l}(\mathfrak{M})$. The adjoint dimension of $\mathfrak{L}$ in $\rho$ is customarily denoted by $n_{A}(\rho)$ in physical literature [12], [13].

Since highest adjoint weights are elements in $\mathfrak{Q}^{*}$, it follows from Corollary 2.1 that

$$
n_{A}(\rho) \leqslant \operatorname{dim} \mathfrak{Q}^{*}=n .
$$

As in Humphreys [5], we denote $\langle\alpha, \beta\rangle=2(\alpha, \beta) /(\beta, \beta)$, for $\alpha, \beta$ in $\mathfrak{Q}^{*}$ whenever $(\beta, \beta) \neq 0$. To sharpen (3.1) further, let $\pi=\left\{\alpha_{1}, \ldots, \alpha_{n}\right\}$ be a fundamental system of roots and let $\Omega=\left\{\Lambda_{1}, \Lambda_{2}, \ldots, \Lambda_{n}\right\}$ be the fundamental system of weights of $\mathfrak{G}$ corresponding to $\pi$. Thus $\Omega$ and $\pi$ are dual of each other in the sense that

$$
\left\langle\Lambda_{j}, \alpha_{k}\right\rangle=\delta_{j k}, \quad j, k=1,2, \ldots, n .
$$

Then the highest weight $\Lambda$ of $\mathfrak{G}$ in $\mathfrak{M}$ is expressed as

$$
\Lambda=m_{1} \Lambda_{1}+m_{2} \Lambda_{2}+\cdots+m_{n} \Lambda_{n},
$$

where $m_{1}, \ldots, m_{n}$ are nonnegative integers determined by $\rho$ (or by $\mathfrak{M}$ ) [5, p. 67]. By (3.2) and (3.3) one finds

$$
m_{j}=\left\langle\Lambda, \alpha_{j}\right\rangle, \quad j=1,2, \ldots, n .
$$

Theorem 3.1 (Adjoint Dimension TheOrem). Let $\mathfrak{i}$ be a finite-dimensional simple Lie algebra over an algebraically closed field $F$ of characteristic 0 and let $\rho$ : $\mathfrak{L} \rightarrow \mathrm{gl}(\mathfrak{M})$ be an irreducible representation. Then the number $n^{+}(\rho)$ of nonzero $m_{j}$ 's in (3.3) is equal to the adjoint dimension $n_{A}(\rho)$ of $\mathfrak{L}$.

Proof. Denote $p=n^{+}(\rho)$. Reordering the roots in $\pi$ if necessary, we may assume that $m_{j}=0$ in (3.3) for $p+1 \leqslant j \leqslant n$, so that $\Lambda$ is expressed as $\Lambda=$ $\sum_{j=1}^{p} m_{j} \Lambda_{j}$. Thus by (3.2) we have

$$
\left(\alpha_{j}, \Lambda\right)=0, \quad p+1 \leqslant j \leqslant n .
$$

Let $\Gamma$ be the highest adjoint weight of any element in $V_{\rho}(\mathfrak{L}, \mathfrak{W})$. Since $\Omega$ is a basis for $\mathfrak{Q}^{*}$ by (3.2), we have

$$
\Gamma=\sum_{j=1}^{n} c_{j} \Lambda_{j}, \quad c_{j} \in F .
$$

In view of (3.5), Lemma 2.2 implies that $\left(\alpha_{j}, \Gamma\right)=0$ for $p+1 \leqslant j \leqslant n$, and this with (3.2) and (3.6) gives $c_{j}=0, p+1 \leqslant j \leqslant n$. Thus it follows from Corollary 2.1 that $n_{A}(\rho) \leqslant p=n^{+}(\rho)$.

Next, we show that $n_{A}(\rho) \geqslant n^{+}(\rho)$ so that we have $n_{A}(\rho)=n^{+}(\rho)$. For every $j$, $1 \leqslant j \leqslant n$, choose an irreducible representation $\rho_{j}$ of $\mathfrak{L}$ in some vector space $\mathfrak{M}_{j}$ with highest weight $m_{j} \Lambda_{j}$ and consider the tensor product $\rho^{\prime}=\rho_{1} \otimes \cdots \otimes \rho_{n}$, a representation of $\mathfrak{L}$ in $\mathfrak{M}^{\prime}=\mathfrak{M}_{1} \otimes \cdots \otimes \mathfrak{M}_{n}$. Then, $\rho^{\prime}$ contains a unique subrepresentation with the highest weight $\Lambda$. We identify this subrepresentation with $\rho$, and the corresponding subspace of $\mathfrak{M}^{\prime}$ with $\mathfrak{M}$. Let $l: \mathfrak{M} \rightarrow \mathfrak{M}^{\prime}$ be the injection and $p$ : $\mathfrak{M}^{\prime} \rightarrow \mathfrak{M}$ be the ( $\mathfrak{Q}$-invariant) projection. Define, for every $j$, a linear mapping $f_{j}$ : $\mathfrak{L} \rightarrow \mathfrak{g I}\left(\mathfrak{M}^{\prime}\right)$ by

$$
f_{j}(x)\left(v_{1} \otimes \cdots \otimes v_{n}\right)=v_{1} \otimes \cdots \otimes \rho_{j}(x) v_{j} \otimes \cdots \otimes v_{n}
$$


for all $x \in \mathfrak{L}, v_{j} \in \mathfrak{M}_{j}, 1 \leqslant j \leqslant n$. Defining a linear mapping $g_{j}: \mathfrak{L} \rightarrow \mathfrak{g l}(\mathfrak{M})$ by

$$
g_{j}(x)=p \circ f_{j}(x) \circ l \text { for } x \in \mathfrak{R}
$$

then $g_{j} \in V_{\rho}(\mathfrak{Q}, \mathfrak{M})$ and the highest adjoint weight of $g_{j}$ is equal to $m_{j} \Lambda_{j}$. This proves $n_{A}(\rho) \geqslant n^{+}(\rho)$.

The authors are indebted to the referee who provided the present proof for the inequality $n_{A}(\rho) \geqslant n^{+}(\rho)$.

Remarks. (1) Notice that (3.1) is also a consequence of Theorem 3.1.

(2) Let $\mathfrak{Q}$ be a Lie algebra over $F$ with basis $x_{1}, x_{2}, \ldots, x_{N}$. An ordered $N$-tuple $t=\left(t_{1}, \ldots, t_{N}\right)$ of elements $t_{1}, \ldots, t_{N}$ in $U(\mathfrak{L})$ is called an adjoint operator of $\mathfrak{L}$ if it satisfies $\left[x_{\mu}, t_{\nu}\right]=\sum_{\lambda=1}^{N} f_{\mu \nu}^{\lambda} t_{\lambda}$, where $f_{\mu \nu}^{\lambda} \in F$ are the structure constants of $\mathfrak{R}$. Denote by $V(\mathfrak{L})$ the space of adjoint operators of $\mathfrak{L}$ and by 3 the center of $U(\mathfrak{L})$. Then $\left(z t_{\mu}\right) \in V(\mathfrak{Q})$ for $z \in \mathbb{3},\left(t_{\mu}\right) \in V(\mathfrak{L})$. Let $K$ be the quotient field of 3 and let $V(\mathfrak{Q})_{K}=K \otimes_{3} V(\mathfrak{L})$ be the scalar extension of $V(\mathfrak{L})$ to $K$. When $\mathfrak{L}$ is a simple Lie algebra over the complex field, it can be shown from the result in [13] that $\operatorname{dim} V(\mathfrak{Q})_{K} \geqslant \operatorname{rank} \mathfrak{R}$ for the algebra $\mathfrak{Q}$ of type $A_{n}, B_{n}, C_{n}, D_{n}$, or $G_{2}$. It has been conjectured in [15] that $\operatorname{dim} V(\mathfrak{R})_{K}=$ rank $\mathfrak{Q}$ over a field of characteristic 0 .

If $\rho=\mathrm{ad}$ is the adjoint representation of $\mathfrak{L}$, the adjoint dimension theorem is strengthened to

THEOREM 3.2. Let $\mathfrak{Q}$ be the same as in Theorem 3.1. Then the adjoint dimension of $\mathfrak{L}$ in the adjoint representation is 1 or 2.

Proof. If $\left\{x_{1}, x_{2}, \ldots, x_{N}\right\}$ is a basis for $\mathfrak{L}$, it is clear that $\left(\operatorname{ad} x_{\mu}\right)$ is an adjoint operator in $V_{0}(\mathfrak{L})$. Thus $\operatorname{dim} V_{0}(\mathfrak{I}) \geqslant 1$. Let $\pi=\left\{\alpha_{1}, \alpha_{2}, \ldots, \alpha_{n}\right\}$ be a fundamental system of roots. Let $\Lambda_{0}$ be the highest weight in ad (relative to $\pi$ ) and $\Omega=\left\{\Lambda_{1}, \ldots, \Lambda_{n}\right\}$ be the fundamental system of weights corresponding to $\pi$. Then

$$
\Lambda_{0}=m_{1} \Lambda_{1}+m_{2} \Lambda_{2}+\cdots+m_{n} \Lambda_{n},
$$

where $m_{j}=\left\langle\Lambda_{0}, \alpha_{j}\right\rangle$ are nonnegative integers. Since $\Lambda_{0}$ is a positive root, the value of $m_{j}=0,1,2$, or 3 . In order to derive a more stringent condition on $m_{j}$, we proceed as follows.

From (3.7), we find

$$
2=\sum_{j=1}^{n} m_{j}\left\langle\Lambda_{j}, \Lambda_{0}\right\rangle
$$

Moreover, $\left\langle\Lambda_{j}, \Lambda_{0}\right\rangle(j=1,2, \ldots, n)$ are easily shown to be strictly positive integers so that (3.8) gives

$$
\sum_{j=1}^{n} m_{j} \leqslant 2
$$

Hence by (3.7)

$$
\Lambda_{0}=\Lambda_{i}, \quad \Lambda_{0}=2 \Lambda_{j}, \quad \text { or } \Lambda_{0}=\Lambda_{k}+\Lambda_{l}
$$

Thus $n^{+}(\mathrm{ad})=1$ or 2 and by Theorem $3.1 n_{A}(\mathrm{ad})=1$ or 2 .

Theorem 3.2 has been proved in [15] for the complex field via a somewhat lengthy process which does not invoke representation theory. It is possible to give a 
more explicit description of (3.10). Utilizing (3.10) and the expression of the fundamental weights $\Lambda_{i}$ in terms of simple roots $\alpha_{j} \in \pi[5$, p. 69], one can express $\Lambda_{0}$ more explicitly in terms of $\Lambda_{i}$ 's as

$$
\Lambda_{0}= \begin{cases}\Lambda_{1}+\Lambda_{n}, & \text { type } A_{n}(n \geqslant 1), \\ \Lambda_{2}, & \text { types } B_{n}(n \geqslant 3) \text { and } D_{n}(n \geqslant 4), \\ 2 \Lambda_{1}, & \text { type } C_{n}(n \geqslant 2), \\ \Lambda_{1}, & \text { types } G_{2}, F_{4}, E_{7} \text { and } E_{8}, \\ \Lambda_{6}, & \text { type } E_{6} .\end{cases}
$$

Here, we used a suitable lexicographical ordering of roots determined by $\pi$ as is specified in [12].

COROllary 3.1. Let $\mathfrak{Z}$ be as in Theorem 3.1. The adjoint dimension of $\mathfrak{L}$ in the adjoint representation is 2 for the case of $\mathfrak{L}=A_{n}(n \geqslant 2)$ and 1 for all other cases.

REMARK. The result of Corollary 3.1 agrees with those by Djokovic [4] and by Krämer [8] who computed directly the multiplicity of the adjoint representation ad contained in a reduction of a tensor product ad $\otimes$ ad by means of the Steinberg formula or its variation. Also, the result of (3.11) as well as Corollary (3.1) is already noted and utilized by Kac [7, pp. 26 and 44] for classification of simple Lie super algebras.

4. Flexible Lie-admissible algebras. Let $\mathfrak{A}$ be a flexible Lie-admissible algebra over a field $F$. Denote by $L_{x}$ and $R_{x}$ the left and right multiplications by $x$ in $\mathfrak{A}$, i.e.,

$$
L_{x} y=x y, \quad R_{x} y=y x, \quad x, y \in \mathfrak{A} .
$$

Let ad and $\theta$ be the linear mappings: $\mathfrak{A} \rightarrow \mathfrak{g l}(\mathfrak{A})$ defined by

$$
\text { ad } x=L_{x}-R_{x}, \quad \theta(x)=L_{x}+R_{x} .
$$

The following result is proved in [10] and is useful for our discussion.

THEOREM 4.1. Let $\mathfrak{A}$ be an algebra over a field $F$ of characteristic $\neq 2$. A necessary and sufficient condition that $\mathfrak{A}$ is flexible Lie-admissible is to have

$$
\begin{aligned}
& \operatorname{ad}[x, y]=[\operatorname{ad} x, \operatorname{ad} y], \\
& \theta([x, y])=[\operatorname{ad} x, \theta(y)]
\end{aligned}
$$

for $x, y \in \mathfrak{A}$.

Therefore, $\mathfrak{A}$ is flexible Lie-admissible if and only if ad and $\boldsymbol{\theta}$ are adjoint operators in $V_{0}\left(\mathfrak{A}^{-}\right)$. Also, (4.2) and Theorem 4.1 lead to

Definition 4.1. Let $\mathfrak{Z}$ be a Lie algebra over $F$. An adjoint operator $S \in V_{0}(\mathfrak{L})$ is said to be symmetric or skew-symmetric if $S$ satisfies

$$
S(x) y=S(y) x \quad \text { or } \quad S(x) y=-S(y) x
$$

for $x, y \in \mathfrak{R}$.

In view of (4.2) and Theorem 4.1, we see that if $\mathfrak{A}$ is flexible Lie-admissible then $\theta$ is symmetric while ad is skew-symmetric in $V_{0}\left(\mathfrak{U}^{-}\right)$. 
THEOREM 4.2. Let $\mathfrak{L}$ be a Lie algebra with product $[x, y]$ over $F$ of characteristic $\neq 2$. For a symmetric element $S$ in $V_{0}(\mathfrak{L})$, let $\mathfrak{L}_{S}$ be an algebra defined on the vector space $\mathfrak{R}$ but with multiplication given by

$$
x y=\frac{1}{2}\{S(x) y+[x, y]\} \text {. }
$$

Then $\mathfrak{L}_{S}$ is flexible Lie-admissible such that $\mathfrak{R}_{\bar{S}}^{-}$is isomorphic to $\mathfrak{R}$. Moreover, $S(x)=\theta(x)$ in $\mathfrak{R}_{s}$.

Proof. Let $L_{x}$ and $R_{x}$ be the left and right multiplications by $x$ in $\mathfrak{L}_{s}$. Then $\operatorname{ad}_{\mathfrak{R}_{s}} x=L_{x}-R_{x}$ and clearly $\operatorname{ad}_{\mathfrak{R}_{s}}=$ ad since $S$ is symmetric. Hence $\mathfrak{L}_{S}$ is Lie-admissible such that $\mathfrak{R}_{S}^{-}=\mathfrak{R}$, and $S=\theta$ in $\mathfrak{R}_{S}$. Noting that

$$
\begin{aligned}
& L_{x} R_{x}=\frac{1}{4}\{S(x)+\operatorname{ad} x\}\{S(x)-\operatorname{ad} x\}, \\
& R_{x} L_{x}=\frac{1}{4}\{S(x)-\operatorname{ad} x\}\{S(x)+\operatorname{ad} x\},
\end{aligned}
$$

we have $L_{x} R_{x}-R_{x} L_{x}=\frac{1}{2}[\operatorname{ad} x, S(x)]=\frac{1}{2} S([x, x])=0$ and hence $\mathfrak{L}_{S}$ is flexible.

Because of Theorems 4.1 and 4.2, the classification of flexible Lie-admissible algebras $\mathfrak{A}$ over a field of $F$ characteristic $\neq 2$ is equivalent to finding all symmetric elements in $V_{0}\left(\mathfrak{U}^{-}\right)$. In the following we complete this classification when $\mathfrak{U}^{-}$is a semisimple Lie algebra over an algebraically closed field of characteristic 0 .

THEOREM 4.3. Let $\mathfrak{A}$ be a finite-dimensional flexible Lie-admissible algebra over an algebraically closed field $F$ of characteristic 0 such that $\mathfrak{A}^{-}$is a simple Lie algebra. Then either $\mathfrak{A}$ is itself a Lie algebra isomorphic to $\mathfrak{A}^{-}$or $\mathfrak{A}^{-}$is a simple Lie algebra of type $A_{n}(n \geqslant 2)$. In the latter case, $\mathfrak{A}$ is either a Lie algebra or isomorphic to an algebra with multiplication given by

$$
x * y=\mu x y+(1-\mu) y x-\operatorname{Tr}(x y) I /(n+1),
$$

which is defined on the space of $(n+1) \times(n+1)$ traceless matrices over $F$, where $x y$ is the matrix product, $\mu \neq \frac{1}{2}$ is a fixed scalar in $F$ and $I$ is the unit matrix.

Proof. We first note that ad is a nonzero skew-symmetric element in $V_{0}\left(\mathfrak{A}^{-}\right)$. Hence, if $\mathfrak{A}^{-}$is not of type $A_{n}(n \geqslant 2)$ then, by Corollary 3.1, there is no nonzero symmetric element in $V_{0}\left(\mathfrak{A}^{-}\right)$. Therefore, $\theta=0$ and $\mathfrak{A}$ is isomorphic to $\mathfrak{U}^{-}$by Theorem 4.1.

Next, suppose that $\mathfrak{U}^{-}$is a Lie algebra of type $A_{n}(n>2)$. We identify $\mathfrak{A}^{-}$with $\mathfrak{L} \equiv \mathfrak{I l}(n+1, F)$. Define the mapping $S: \mathfrak{L} \rightarrow \mathfrak{g l}(\mathfrak{L})$ by

$$
S(x) y=x y+y x-\frac{2}{n+1}(\operatorname{Tr} x y) I,
$$

where $x y$ indicates the matrix product and $I$ is the $(n+1) \times(n+1)$ identity matrix. Since the right side of (4.6) has trace $0, S$ is well defined. It is easily checked that $S$ is a symmetric element in $V_{0}(\mathfrak{L})$. Clearly, ad $\neq 0$ and $S \neq 0$, since $x y+y x \notin F I$ for some $x, y \in \mathfrak{L}$ if $n \geqslant 2$. Noting that ad and $S$ are linearly independent, it follows from Corollary 3.1 that any symmetric element in $V_{0}(\mathfrak{L})$ is proportional to $S$ and hence $\theta=\alpha S$ for some $\alpha \in F$. Therefore, by Theorems 4.1 
and 4.2 we conclude that $\mathfrak{A}$ is isomorphic to an algebra defined on $\mathfrak{L}$ but with multiplication given by

$$
\begin{aligned}
x * y & =\frac{1}{2}\{\alpha S(x)+\operatorname{ad} x\} y \\
& =\frac{1}{2}\left\{(\alpha+1) x y+(\alpha-1) y x-\frac{2 \alpha}{n+1}(\operatorname{Tr} x y) I\right\} .
\end{aligned}
$$

If $\alpha=0$ then $\mathfrak{A}$ is isomorphic to $\mathfrak{U}^{-}=\mathfrak{R}$. If $\alpha \neq 0$ then, dividing both sides of (4.7) by $\alpha$ and setting $\mu=\frac{1}{2}(1+1 / \alpha) \neq \frac{1}{2}$, we obtain the algebra described by (4.5).

This classification includes the pseudo-octonion algebra introduced in [14] as a special case. The proof of Theorem 4.3 together with Corollary 3.1 yields

COROllaRY 4.1. Let $\mathfrak{Q}$ be a simple Lie algebra over an algebraically closed field of characteristic 0 . Then any skew-symmetric element in $V_{0}(\mathfrak{L})$ is proportional to ad. The Lie algebra of type $A_{n}(n \geqslant 2)$ alone has a nonzero symmetric element in $V_{0}(\mathfrak{L})$.

Corollary 4.1 reproduces the result in [12], [4] and [8]. Also, the restriction of characteristic 0 can be relaxed for the uniqueness of skew-symmetric elements in $V_{0}(\mathfrak{R})$ (see [15]). However, the present weaker form is sufficient for our purpose. Also, it is related to a fact that only the Lie algebra $A_{n}(n \geqslant 2)$ has a 3 rd order Casimir invariant ([2], [12] and [19]).

Let $\mathfrak{A}$ be a flexible Lie-admissible algebra over a field of characteristic $\neq 2$ such that $\mathfrak{A}^{-}$is a direct sum of simple Lie algebras $\mathfrak{U}_{i}$. It is shown in [11] that each $\mathfrak{A}_{i}$ is an ideal of $\mathfrak{A}$ and so $\mathfrak{A}$ is the direct sum of simple flexible Lie-admissible algebras $\mathfrak{U}_{i}$. Thus as an immediate consequence of Theorem 4.3 we have

COROllary 4.2. Let $\mathfrak{A}$ be a finite-dimensional flexible Lie-admissible algebra over an algebraically closed field of characteristic 0 such that $\mathfrak{A}^{-}$is a semisimple Lie algebra. Then $\mathfrak{A}$ is the direct sum of simple Lie algebras and simple algebras defined by (4.5).

Corollary 4.2 essentially completes the classification of flexible Lie-admissible algebras in the characteristic zero case which was proposed by Albert [1] in 1948 . As an application of Theorem 4.3 one can easily prove the following result of Laufer and Tomber [9].

CoROllaRy 4.3. Let $\mathfrak{A}$ be a finite-dimensional power-associative flexible Lie-admissible algebra over a field $F$ of characteristic 0 such that $\mathfrak{U}^{-}$is semisimple. Then $\mathfrak{A}$ is a Lie algebra isomorphic to $\mathfrak{A}^{-}$.

Proof. We may assume that $F$ is algebraically closed since any scalar extension of $\mathfrak{U}^{-}$is semisimple also. Due to Corollary 4.2 it is sufficient to show that the algebra defined by (4.5) cannot be power-associative. Let $\mathfrak{U}^{-}=\mathfrak{g l}(n+1, F)$ with $n \geqslant 2$ and let $\mathfrak{A}$ have the multiplication given by (4.5). If $\mathfrak{A}$ is power-associative, it is easily checked that $(x * x) *(x * x)=[(x * x) * x] * x$ implies

$$
\left(\operatorname{Tr} x^{2}\right) x^{2}-\left(\operatorname{Tr} x^{3}\right) x-\frac{1}{n+1}\left[\operatorname{Tr}\left(x^{2}\right)\right]^{2} I=0
$$

for all $x \in \mathfrak{A}$. This is absurd since, for example, the diagonal matrix $x=$ $\operatorname{diag}\{1,2,-3,0, \ldots, 0\}$ does not satisfy (4.8). Thus $\mathfrak{A}$ cannot be power-associative. 
For a later purpose we simply quote here the following well-known theorem [5, p. 118].

TheOREM 4.4. Let $\mathfrak{L}$ and $F$ be the same as in Corollary 4.1. Then any bilinear form (, ) on $\mathbb{R}$ satisfying the invariant condition

$$
([x, y], z)=(x,[y, z]) \text { for } x, y \in \mathfrak{L}
$$

must be of the form $(x, y)=\alpha \operatorname{Tr}(\operatorname{ad} x$ ad $y)$ for some $\alpha \in F$.

5. The reductive case. A finite-dimensional Lie algebra $\mathfrak{L}$ over a field $F$ of characteristic 0 is called reductive if the adjoint representation of $\mathfrak{L}$ is completely reducible. This is equivalent to the fact that $\mathfrak{L}$ is the direct sum of a semisimple Lie algebra and an abelian Lie algebra, or the solvable radical of $\mathfrak{L}$ is the center of $\mathfrak{L}[\mathbf{3}$, p. 56]. Thus if $\mathfrak{Z}$ is reductive then $[\mathfrak{Z}, \mathfrak{Q}]$ is the unique Levi-factor of $\mathfrak{L}$.

The classification in $\$ 4$ can be applied to determine all flexible Lie-admissible algebras $\mathfrak{A}$ such that $\mathfrak{A}^{-}$is reductive and the Levi-factor of $\mathfrak{A}^{-}$is simple. Thus, let

$$
\mathfrak{A}^{-}=\Im \oplus \mathbb{Z}
$$

be the direct sum of a simple Lie algebra $\subseteq$ and the center 3 of $\mathfrak{U}^{-}$. Henceforth we denote the elements of $\subseteq$ by $x, y, z, \ldots$ while those of 3 are labelled by $a, b$, $c, \ldots$.

THEOREM 5.1. Let $\mathfrak{A}$ be a finite-dimensional flexible Lie-admissible algebra over an algebraically closed field $F$ of characteristic 0 such that $\mathfrak{U}^{-}$is a reductive Lie algebra with the Levi-decomposition given by (5.1). If the Levi-factor $\subseteq$ of $\mathfrak{A}^{-}$is simple then $\mathfrak{A}$ is isomorphic to an algebra defined on the vector space $\subseteq \oplus 3$ with multiplication given by

$$
\begin{aligned}
& x * y=\theta_{\Im}(x) y+[x, y]+[\operatorname{Tr}(\operatorname{ad} x \operatorname{ad} y)] c, \\
& x * a=a * x=\tau(a) x, \\
& a * b=b * a \in 3,
\end{aligned}
$$

where $\theta_{\Im}$ is a symmetric element in $V_{0}(\Im), x, y \in \subseteq, a, b \in 3, c$ is fixed in 3 , and $\tau$ is a linear functional on 3 .

Proof. In view of Theorems 4.1 and 4.2, it suffices to determine the general form of symmetric elements in $V_{0}\left(\mathfrak{U}^{-}\right)$. Let $\mathfrak{L}=\mathfrak{A}^{-}$. Since $\operatorname{Hom}_{F} \mathfrak{L}=\operatorname{Hom}_{F} \mathfrak{S} \oplus$ $\operatorname{Hom}_{F}(\Im, 3) \oplus \operatorname{Hom}_{F}(3, \Im) \oplus \operatorname{Hom}_{F} 3$, it is convenient to express a linear mapping $\theta: \mathfrak{Z} \rightarrow$ Hom $\mathfrak{Z}$ by the matrix notation

$$
\theta=\left(\begin{array}{ll}
A & B \\
G & D
\end{array}\right), \quad \theta(t)=\left(\begin{array}{ll}
A(t) & B(t) \\
G(t) & D(t)
\end{array}\right)
$$

for $t \in L$ where $A, G, B, D$ are the component linear mappings of $\theta$ from $\mathfrak{L}$ into $\operatorname{Hom} \Im, \operatorname{Hom}(\Im, 3), \operatorname{Hom}(3, \Im), \operatorname{Hom} 3$, respectively. In particular,

$$
\operatorname{ad} x=\left(\begin{array}{cc}
\operatorname{ad}_{\Im} x & 0 \\
0 & 0
\end{array}\right), \quad x \in \subseteq,
$$

while ad $a=0$ for $a \in Z$, since $Z$ is the center of $\mathfrak{L}$. 
Suppose now that $\theta$ is a symmetric element in $V_{0}(\mathfrak{L})$. Equating $0=\theta([x, a])=$ [ad $x, \theta(a)]$ with (5.5) and (5.6), we have

$$
\left(\operatorname{ad}_{\Im} x\right) A(a)=A(a) \operatorname{ad}_{\Im} x, \quad\left(\operatorname{ad}_{\Im} x\right) B(a)=0, \quad G(a) \operatorname{ad}_{\Im} x=0 .
$$

Since $\mathrm{ad}_{\mathfrak{S}}$ is an irreducible representation of $\mathfrak{S}$, by Schur's lemma these relations demand that

$$
\begin{aligned}
& A(a)=\tau(a) I_{\Im}, \\
& B(a)=G(a)=0,
\end{aligned}
$$

where $I_{\mathfrak{S}}$ is the identity mapping on $\subseteq$ and $\tau$ is a linear functional on 3 . Hence (5.5) and (5.7) give

$$
\theta(a)=\left(\begin{array}{cc}
\tau(a) I_{\Im} & 0 \\
0 & D(a)
\end{array}\right)
$$

while $\theta([a, b])=[\operatorname{ad} a, \theta(b)]$ is an identity. The symmetric condition $\theta(x) a=\theta(a) x$ with (5.5) leads to $B(x) a=A(a) x$ and $D(x) a=G(a) x$, which reduce to

$$
\begin{aligned}
D(x) & =0, \\
B(x) a & =\tau(a) x
\end{aligned}
$$

by (5.7). Similarly, the relations $\theta([x, y])=[\operatorname{ad} x, \theta(y)]$ and $\theta(x) y=\theta(y) x$ imply

$$
\begin{aligned}
A([x, y]) & =[\operatorname{ad} x, A(y)], \\
B([x, y]) & =\left(\operatorname{ad}_{\subseteq} x\right) B(y), \\
G([x, y]) & =-G(y) \operatorname{ad}_{\subseteq} x, \\
A(x) y & =A(y) x, \\
G(x) y & =G(y) x .
\end{aligned}
$$

Denote by $\theta_{\subseteq}$ the restriction of $A$ to $\subseteq$. It then follows from (5.10a) and (5.11a) that $\theta_{\Im}$ is a symmetric element in $V_{0}(\Im)$. Similarly, using $\theta(a) b=\theta(b) a$, we argue that the restriction of $D$ to 3 is a symmetric element $\theta_{3}$ in $V_{0}(3)$. We also have from (5.10c)

$$
G([x, y]) z=-G(y)[x, z] .
$$

Since 3 is abelian, 8 has a symmetric nondegenerate bilinear form ( , ). Noting that $G: \mathfrak{R} \rightarrow \operatorname{Hom}(\subseteq, 3)$, one can define a bilinear form $(,)_{a}$ on $\subseteq$ by

$$
(x, y)_{a} \equiv(a, G(x) y) .
$$

Then (5.12) implies $([x, y], z)_{a}=-(y,[x, z])_{a}$, so that $(,)_{a}$ is an invariant form on ऽ. Therefore, Theorem 4.4 assures that

$$
(x, y)_{a}=\alpha(a) \operatorname{Tr}(\operatorname{ad} x \operatorname{ad} y)
$$

for some $\alpha(a) \in F$. Comparing (5.14) with (5.13), we see that $\alpha$ is a linear functional on 3 . Since (, ) is nondegenerate on 3 , we have $\alpha(a)=(a, c)$ for some $c \in 3$. Again, by the nondegeneracy of (, ), this together with (5.13) and (5.14) implies

$$
G(x) y=[\operatorname{Tr}(\operatorname{ad} x \operatorname{ad} y)] c .
$$


Then (5.11b) is an identity. We summarize these results as

$$
\begin{aligned}
& \theta(x) y=A(x) y+G(x) y=\theta_{\Im}(x) y+[\operatorname{Tr}(\operatorname{ad} x \operatorname{ad} y)] c, \\
& \theta(x) a=G(a) x=B(x) a=\tau(a) x \\
& \theta(a) b=\theta(b) a=\theta_{3}(a) b \in 3 .
\end{aligned}
$$

In view of Theorems 4.1 and $4.2,(5.15),(5.16)$ and (5.17) respectively give the multiplications given by (5.2), (5.3) and (5.4).

Notice that $\theta_{\subseteq}$ has been determined by Theorem 4.3. Thus if $\subseteq$ is not of type $A_{n}$ $(n \geqslant 2)$ then $\theta_{\Im}=0$. If $\subseteq$ is of type $A_{n}(n \geqslant 2)$ then by Theorem 4.3 (5.2) is explicitly written as

$$
x * y=\mu x y+(1-\mu) y x-\frac{1}{n+1} \operatorname{Tr}(x y) I+[\operatorname{Tr}(\operatorname{ad} x \operatorname{ad} y)] c,
$$

where we identified $\subseteq=\mathfrak{s l}(n+1, F), x y$ denotes the matrix product, and $\mu \neq \frac{1}{2}$ is in $F$. In both cases, the remaining multiplication is described by (5.3) and (5.4).

The classification in Theorem 5.1 includes the quaternion algebras $\mathfrak{Q}$ as well as the general linear algebra $\operatorname{gl}(n, F)$ where the Levi-factor $\subseteq$ of $\mathfrak{Q}^{-}$and $\operatorname{gl}(n, F)^{-}$ are respectively $\mathfrak{g l}(2, F)$ and $\mathfrak{g l}(n, F)$, and $\mathfrak{g l}(n, F)$ has one-dimensional center $F I$. A less obvious example arises from the pseudoquaternion algebra $\mathfrak{B}_{4}$ introduced in [14]. The algebra $\mathfrak{B}_{4}$ is a 4-dimensional algebra over $F$ with basis $\left\{e, x_{1}, x_{2}, x_{3}\right\}$ such that

$$
\begin{gathered}
x_{j} x_{k}=\sum_{l=1}^{3} \varepsilon_{j k l} x_{l}+\delta_{j k} e, \\
x_{j} e=e x_{j}=x_{j}, \quad e e=-e, \quad j=1,2,3,
\end{gathered}
$$

where $\varepsilon_{j k l}$ is the antisymmetric Levi-Civita symbol. $\mathfrak{B}_{4}$ is not associative but flexible Lie-admissible, and $\mathfrak{P}_{4}^{-}$is reductive with Levi-factor $\mathfrak{g l}(2, F)$ and center $F e$. Notice also $x y+y x=\operatorname{Tr}(x y) I$ for $x, y \in \mathfrak{I l}(2, F)$.

ACKNOWLEDGEMENT. The authors would like to express their gratitude to the referee for many invaluable suggestions, especially for providing the proof of $n_{A}(\rho) \geqslant n^{+}(\rho)$ in Theorem 3.1 .

\section{REFERENCES}

1. A. A. Albert, Power-associative rings, Trans. Amer. Math. Soc. 64 (1948), 552-597.

2. A. Borel and C. Chevalley, The Betti numbers of the exceptional groups, Mem. Amer. Math. Soc., no. 14, Amer. Math. Soc., Providence, R. I., 1955, pp. 1-9.

3. N. Bourbaki, Lie groups and Lie algebras. I, Addison-Wesley, Reading, Mass., 1975.

4. D. Ž. Djoković, Classification of some 2-graded Lie algebras, J. Pure Appl. Algebra 7 (1976), 217-230.

5. J. E. Humphreys, Introduction to Lie algebras and representation theory, Grad. Texts in Math., no. 9, Springer-Verlag, Berlin and New York, 1972.

6. N. Jacobson, Lie algebra, Interscience Tracts in Pure and Appl. Math., no. 10, Interscience, New York, 1962.

7. V. G. Kac, Lie super algebra, Advances in Math. 26 (1977), 8-96.

8. M. Krämer, Eine Klassifikation Bestimmter Untergruppen Kompakter Zusammen-Hängender Lie gruppen, Comm. Algebra 3 (1975), 691-737.

9. P. J. Laufer and M. L. Tomber, Some Lie-admissible algebras, Canad. J. Math. 14 (1962), 287-292.

10. H. C. Myung, Some classes of flexible Lie-admissible algebras, Trans. Amer. Math. Soc. 167 (1972), 79-88. 
11. Lie-admissible algebras, Hadronic J. 1 (1978), 169-193.

12. S. Okubo, Gauge groups without triangular anomaly, Phys. Rev. D(3) 16 (1977), 3528-3534.

13. __ Casimir invariants and vector operators in simple and classical Lie algebras, J. Math. Phys. 18 (1977), 2382-2394.

14. __ Pseudo-quaternion and pseudo-octonion algebras, Hadronic J. 1 (1978), 1250-1278.

15. S. Okubo and H. C. Myung, On the classification of simple flexible Lie-admissible algebras, Hadronic J. 2 (1979), 504-567.

16. L. O'Raifeartaigh, Broken symmetry, Group Theory and its Applications, (E. M. Loebl, ed.), Academic Press, New York, 1968.

17. R. M. Santilli, Lie-admissible approach to the hadronic structure, vol. I, Hadronic Press, Nonantum, Mass., 1978; Vols. II and III (to appear).

18. G. B. Seligman, Modular Lie algebras, Ergebnisse der Math. und ihrer Grenzgebiete, Bd. 40, Springer-Verlag, Berlin and New York, 1967.

19. G. Warner, Harmonic analysis on semi-simple Lie groups. I, Springer-Verlag, Berlin and New York, 1972.

20. E. P. Wigner, On representations of certain finite groups, Amer. J. Math. 63 (1941), 57-63.

Department of Physics and Astronomy, University of Rochester, Rochester, New York 14627

Department of Mathematics, University of Northern Iowa, Cedar Falls, Iowa 50613 